\title{
Als wäre nichts geschehen
}

S chwerpunkt dieser Ausgabe ist die wegen ihrer vermeintlichen Banalität oft unterschätzte häufigste dermatologische Krankheit, das Kontaktekzem. Insofern sind Arbeiten zur Pathophysiologie des allergischen Kontaktekzems (Enk) sowie zu den verschiedenen, auch differenzialdiagnostischen Aspekten der nicht ekzematösen Kontaktdermatitis (Aberer, Kränke) von besonderem Interesse.

Das berufsbedingte

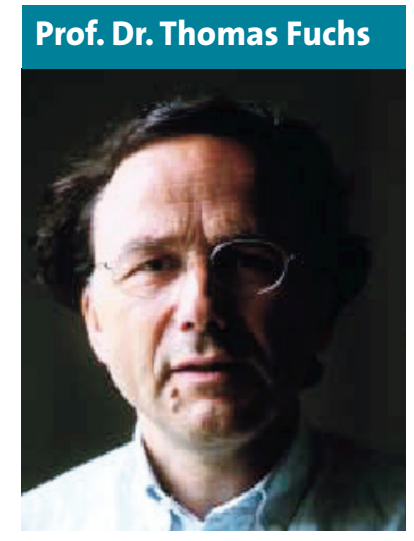

Hautklinik und Poliklinik, Georg-August-Universität, Göttingen, Präsident des ÄDA

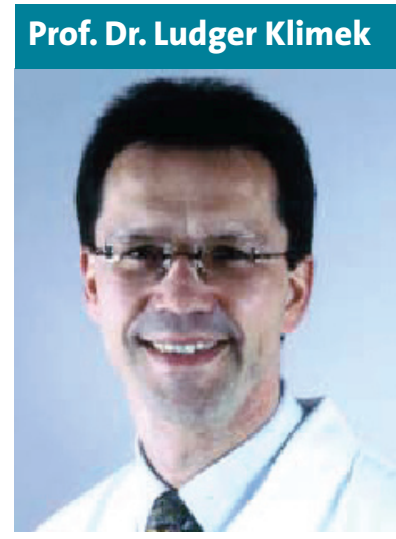

Zentrum für Rhinologie und Allergologie, Wiesbaden, Beirat im Vorstand des ÄDA
Handekzem ist, wie schon

seit Jahren, trotz vielfältiger präventiver Maßnahmen und Projekte, weiterhin von erheblicher psychosozialer und sozioökonomischer Bedeutung. Es ist gerade so, als wäre nichts geschehen. Die BK 5101 rangiert immer noch auf Platz eins der gemeldeten BK-Ziffern, weit vor den Wirbelsäulenerkrankungen und der Lärmschwerhörigkeit.

Bei den Betroffenen führt die Erkrankung meist zu einem einschneidenden Knick in der Lebensplanung, nicht selten geht der Arbeitsplatz verloren und der Beruf muss aufgegeben werden. Dies wiegt besonders schwer, wenn dem Arbeitnehmer als Folge der so genannten weißen Meldung gekündigt wird, ohne dass \$3-Maßnahmen angedacht und schon gar nicht ausgeschöpft wurden. Skudlik und Schwanitz unterstreichen in ihrem Beitrag die Wichtigkeit verschiedener Präventionsformen.

Eine interessante Frage ist in diesem Zusammenhang, ob jenseits der Statistiken die Naturgummilatexsensibilisierung tatsächlich abgenommen hat. Die offiziellen Zahlen der BGW erwecken den Eindruck, dass es so sein könnte. Zweifel sind angebracht. Denn auf Wunsch der Arbeitnehmer unterbleiben viele Verdachtsmeldungen, um nur ja nicht den Arbeitsplatz zu gefähr- den. Das gilt besonders für den pflegerischen und ärztlichen Bereich. Misstrauen gegen offizielle Statistiken und Wachsamkeit sind also angebracht.

Anders als das Maurerekzem kann die beruflich verursachte Naturgummilatexallergie nicht vorhersehbare lebensbedrohliche Reaktionen bei banalen gynäkologischen oder zahnärztlichen Untersuchungen auslösen. Die Betroffenen fallen bislang durch die Maschen des „Systems“. Denn ihre Krankheit wird versicherungsrechtlich (un)verständlicherweise nur unzureichend berücksichtigt. Ideal wäre es, der Komplexität des Krankheitsbildes mit einer neuen BK-Ziffer zu begegnen - vorstellbar ist eine Kombination der BK 5101 und der 4301.

Mit mangelhafter ärztlicher Versorgung hat eine weitere Arbeit in diesem Heft zu tun. Die in dieser Form in Deutschland noch nicht durchgeführte Untersuchung von Nolting et al. ist von

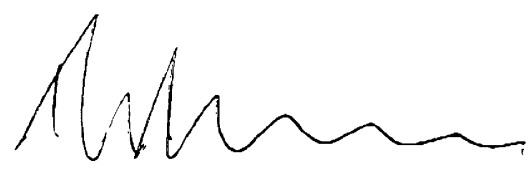

Prof. Dr. Thomas Fuchs enormer sozialpolitischer Bedeutung. Hiernach beendeten mehr als zwei Drittel von fast 7.000 Patienten mit allergischen Atemwegserkrankungen ihre längere und unbefriedigend verlaufene Patientenkarriere in der Primärversorgung auf eigene Initiative und wechselten zum Facharzt. Der Wechsel in die spezialisierte allergologische Versorgung führte zu einer Optimierung nicht nur der symptomatischen Therapie, sondern vor allen Dingen zur erstmaligen Verschreibung einer spezifischen Immuntherapie. Wesentlicher Punkt ist die schon immer vermutete und hier weitgehend bestätigte Tatsache, dass Patienten vielfach nicht oder erst zu spät zu einem allergologischen Spezialisten überwiesen werden. In der lesenswerten Arbeit wird die Befürchtung ausgesprochen, dass die Fortschritte der letzten Jahre in der Allergologie und Immunologie an den Primärärzten mehr oder weniger spurlos vorbeigegangen sind.

Hieraus ließe sich kurz und prägnant ableiten: „Bei Allergien besser gleich zum Spezialisten." Es würde aber völlig reichen, wenn das von einem breiten Konsens getragene Konzept der dreistufigen Versorgung allergischer $\mathrm{Pa}$ tienten in Deutschland endlich umgesetzt würde. Ärgerlicherweise wurde es von den zuständigen Gremien ohne Begründung schubladiert.

Den Leserinnen und Lesern des Allergo Journal wünschen wir in den kommenden Wochen Zeit für besinnliche Stunden und allen Widrigkeiten zum Trotz ein erfolgreiches neues Jahr.
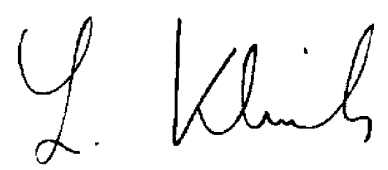

Prof. Dr. Ludger Klimek 